\title{
Simulation Research of Double-shell Flat Block Assembly Line on the Basis of Double-flat Floor Pull-in Method
}

\author{
Wang Yue, HE Lei
}

\begin{abstract}
Rarely can we find documents study on double-flat assembly line on the basis of double-flat floor pull-in method and its simulation research. Thus, this paper present this idea and built a simulation model of double-shell flat floor assembly line by simulation software Plant Simulation and operated this model on the basis of double-flat floor pull-in method. By analysing the outcomes we found blocking situation on several stations. To avoid this problem we use DOE theory to conduct multiple experiments and find a way to change location of buffer stations which can solve this problem. By applying relocated buffer station the blocking situation is well solved.
\end{abstract}

Index Terms - Double-shell flat block assembly line, double-flat floor pull-in method, blocking station, plant simulation.

\section{INTRODUCTION}

For most ship yard there are basically two ways to install floor in panel block, the floor insertion method and the floor pull-in method. Installing longitudinal frames firstly then insert floors into these longitudinal frames vertically by crane is called the floor insertion method and as Fig.1 put floor from one end then pull it in through these longitudinal frames is called the floor pull in method. Lots of studies show the second method can save much more materials and time than the first method ${ }^{[1]}$.

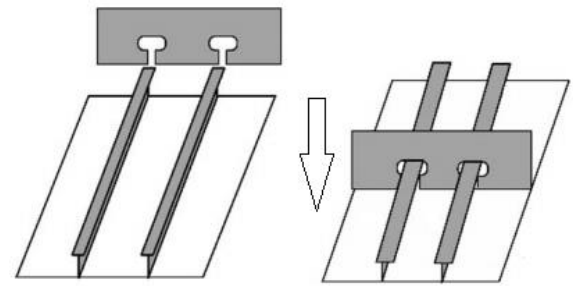

Fig.1 Illustration of floor pull-in method

Double-flat floor pull-in method is developed on the basis of single flat floor pull-in method. Considering the construction of double bottom block, the single flat floor pull-in method can be only applied on the inner panel. While the outer panel of this block is still completed by the traditional floor insertion method. To improving production efficiency and avoiding waste of materials double-flat floor pull-in method is recommended. Procedure of this method is

Wang Yue, Professor, School of Naval Architecture \& Ocean Engineering, Jiangsu University of Science and Technology, Zhenjiang, Jiangsu, China.

HE Lei, Graduate student, School of Naval Architecture \& Ocean Engineering, Jiangsu University of Science and Technology, Zhenjiang, Jiangsu, China. completing welding of longitudinal frames on the outer panel ahead, then using a crane to turn inner panel upside down, which has already installed floors by pull-in method. At last, pull it in through the longitudinal frames of outer panel that prepared ahead.

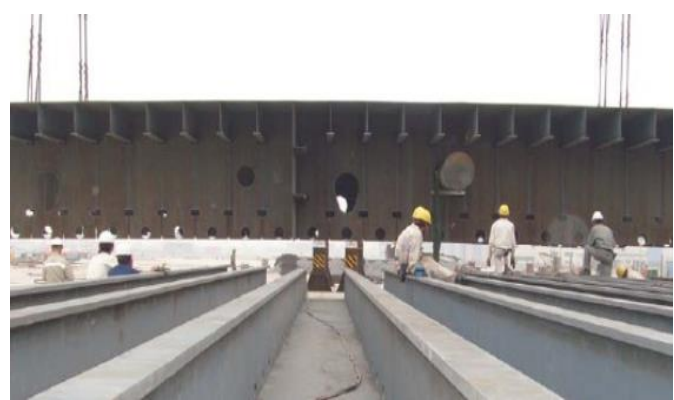

Fig.2 Illustration of double-flat floor pull-in method

To apply this double-flat floor pull-in method, traditional single shell flat block assembly line could waste a lot of time while one panel of this double-shell block completed and waiting another panel of this block just make entry of the same assembly line. Some ship yards have constructed two parallel assembly lines to apply this method, this kind of assembly line is called double-shell flat block assembly line. To examine how good this assembly line works and what is the problem this assembly line could face. In this paper, we simulated this kind of assembly line with the double-flat floor pull-in method. Then we found blocking situation happens in several stations. By analyzing the outcomes of the simulation we put forward a way to avoid the problem by setting buffer stations in right places. According to simulation of our new design, the problem is well solved.

\section{DOUBLE-SHELL FLAT BLOCK ASSEMBLY LINE}

\section{A. Concept of Double-shell flat block assembly line}

Double-shell flat block assembly line is actually formed by two single-shell flat block assembly lines. One of them produce inner panel with welded longitudinal frames before side move station, and completing inner panel block with welded longitudinal frames, floors, longitudinal, pre-outfitting and so on at the end of its assembly that producing it. Another assembly line produce outer panel with welded longitudinal frames. At last, inner and outer panel group the grouping station to become a whole double-shell flat block ${ }^{[2]}$.

No matter which flat block assembly line all of them should consider station setup, worker deployment and equipment location in the real circumstance so to make sure the assembly line work balanced and continually. 


\section{B. Scheme design of double-shell flat block assembly line}

Not every flat panel has to go through all the flat block assembly stations. According to the requirement of production, flat block can be divided into 3 types $^{[3]}$ :

1) Double-shell flat block: Inner panel pass through all the stations and manufacturing procedures including plate welding, floor and longitudinal frames welding, pre-outfitting, welding of other parts and structure ${ }^{[4]}$. Outer panel only go through part of the stations then move out though side moving station with longitudinal frames be welded. At last, both outer panel and inner panel are welded together forming a complete double-shell flat block.

2) Type A single-shell flat block ${ }^{[5]}$ : Only inner panel go through all the stations and manufacturing procedures including plate welding, floor and longitudinal frames welding, pre-outfitting, welding of other parts and structure then becoming a Type A block.

3) Type B single-shell flat block ${ }^{[5]}$ : Only outer panel go through part of the stations then move out though side moving station with longitudinal frames be welded.

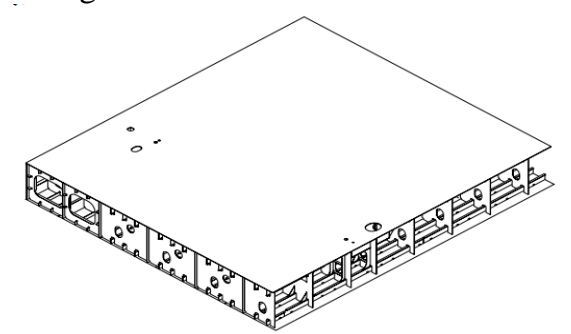

Fig.3 Double-shell block

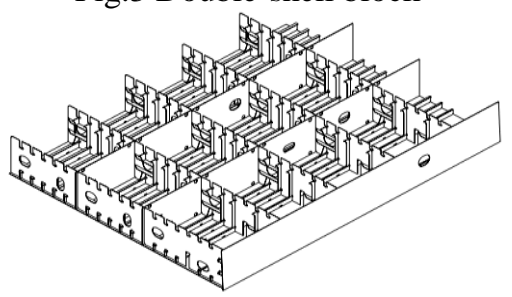

Fig.4 Type A single-shell block

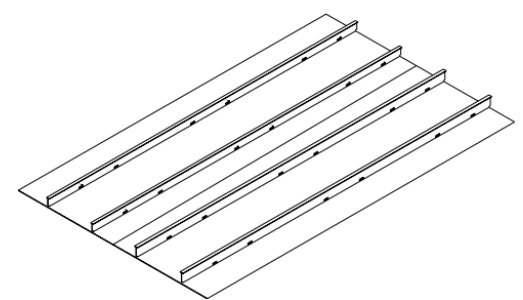

Fig.5 Type B single-shell block

Double-shell flat block assembly line can not only produce Type A: single-shell block and Type B: single-shell block but also can realize manufacturing of double-shell flat block. Its procedure is illustrated as Fig. 6 and Table. 1 below.

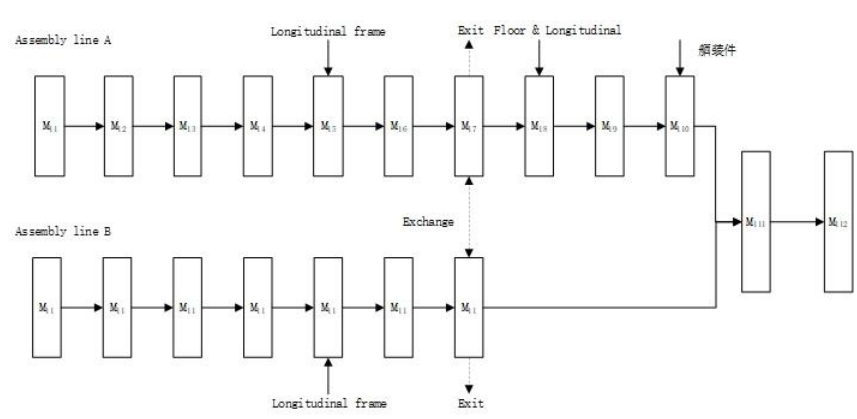

Fig.6 Procedure of double-shell flat block assembly line
Table.1 Meaning of stations in double-shell flat assembly line

\begin{tabular}{cc}
\hline Stations & Functions \\
\hline $\mathrm{M}_{11}, \mathrm{M}_{21}$ & Cutting and line drawing \\
$\mathrm{M}_{12}, \mathrm{M}_{22}$ & Plate welding \\
$\mathrm{M}_{13}, \mathrm{M}_{23}$ & FCB single-face welding \\
$\mathrm{M}_{14}, \mathrm{M}_{24}$ & Checking and buffering \\
$\mathrm{M}_{15}, \mathrm{M}_{25}$ & Longitudinal frame installing \\
$\mathrm{M}_{16}, \mathrm{M}_{26}$ & Longitudinal frame welding \\
$\mathrm{M}_{17}, \mathrm{M}_{27}$ & Side moving \\
$\mathrm{M}_{18}$ & Floor and longitudinal installing \\
$\mathrm{M}_{19}$ & Floor and longitudinal welding \\
$\mathrm{M}_{110}$ & Pre-outfitting \\
$\mathrm{M}_{111}$ & Double-shell grouping and welding \\
$\mathrm{M}_{112}$ & Checking and moving out \\
\hline
\end{tabular}

\section{SIMULATION OF DOUBLE-SHELL FLAT BLOCK ASSEMBLY LINE BASED ON DOUBLE-FLAT FLOOR PULL-IN METHOD}

In this paper, we choose a real double-shell flat block assembly line to simulate based on double-flat floor pull-in method. The assembly line is 330 meters long and 22.5 meters wide. Stations of assembly line A and B is exactly the same from the beginning to the side moving station, and they are connected through side moving station, assuming any stations that before side moving station of line A was broken. The panels of line A can transfer through side moving station from line A to line B to continue the procedure. Store yards are around the assembly line to supply longitudinal frames, floors, longitudinal and outfitting. We operate this simulation model on a monthly schedule. For reason of limited space in simulation software we zoom in the real station size on 10:1 scale, if real length of panel in each station is 20 meters then it will be 2 meters in simulation model. Part of the monthly schedule of working time in each station is shown in Fig.7.

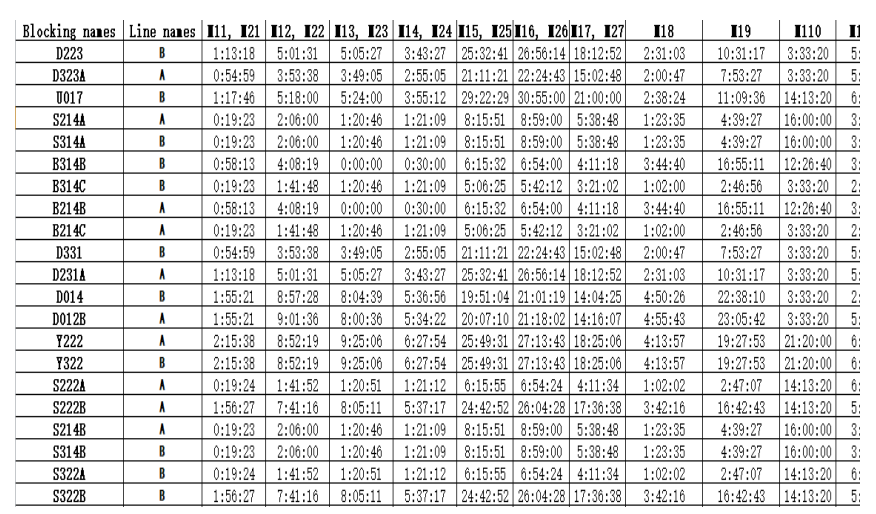

Fig.7 Operation time of each plate on double-shell flat block assembly line based on floor pull-in method

The simulation model built with Plant Simulation is show as below.

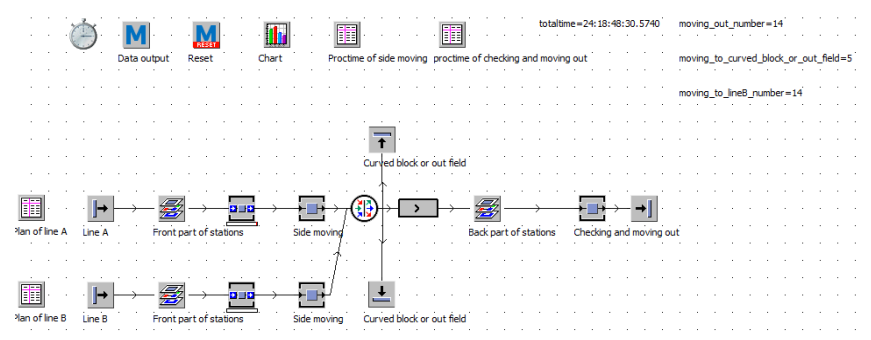

(a) Overview of the assembly line 


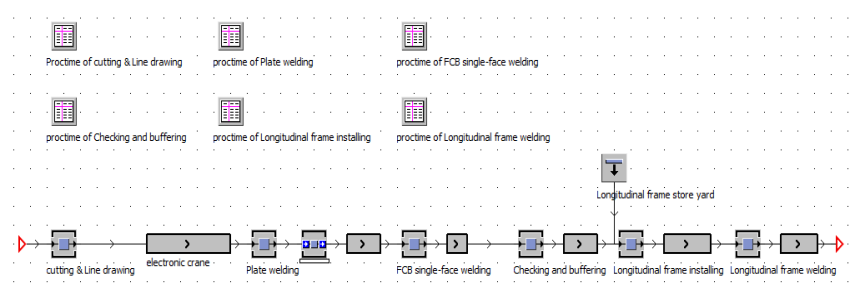

(b) Front part of stations

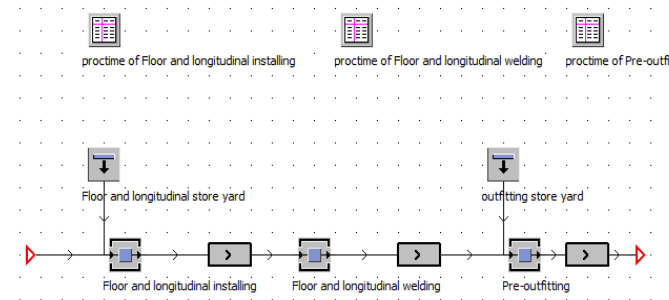

(c) Back part of stations

Fig.8 Double-shell flat block assembly line

After simulation of the monthly schedule from the outcome shown in Fig. 8 we observed that production of 71 different panels cost 24 days 18 hours 48 minutes and 30 seconds.

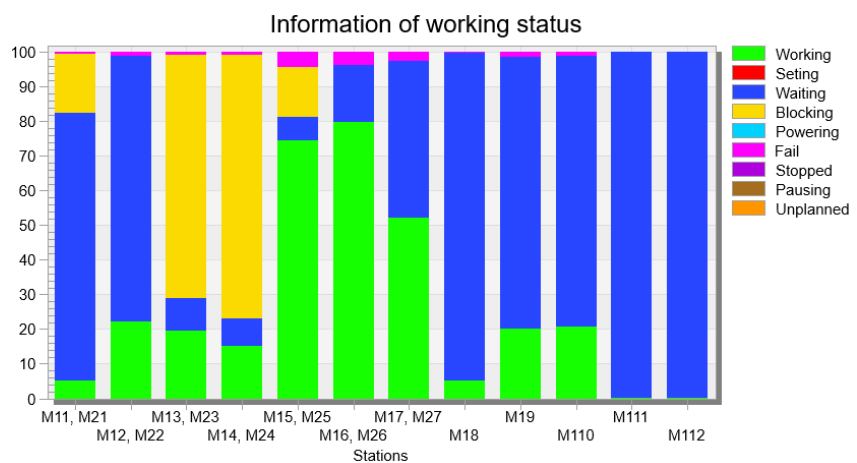

Fig.9 Information of working status of each stations

And Fig.9 shows working status of each stations. Green bar stands for percentage of working time; blue bar stands for percentage of waiting time and yellow bar stands for percentage of blocking time. From this chart we found a problem that FCB single-face welding station and checking station is severely blocked, thus, we suggest setting suitable volume of buffer station behind checking station to lower the percentage of blocking faced by those stations.

\section{WAYS OF AVOIDING STATION BLOCKING}

By analyzing we found that the causing of blocking is long processing time in longitudinal frame installing station. This will lead to panels before this station blocked in the front stations even the job has already done on this station and no panel can go to the longitudinal frame welding station for further processing. The situation is caused by inappropriate station setups, to solve this problem we need to adjust the stations ${ }^{[6]}$ rather than other ways such as personal scheduling. After multiple times of tests we found that adding buffer station in appropriate place can lower blocking percentage of those two stations effectively ${ }^{[7]}$.

\section{A. Design of buffer station}

We set up new buffer station after checking station, which is shown as Fig.10. Then we use experiment manager which based on DOE theory to design experiments that aims at finding the appropriate volume of the buffer station. The value scope is from 1 to 30 , difference value is 1 , we set blocking percentage of FCB single-face welding and checking stations as output value, totally 30 tests.

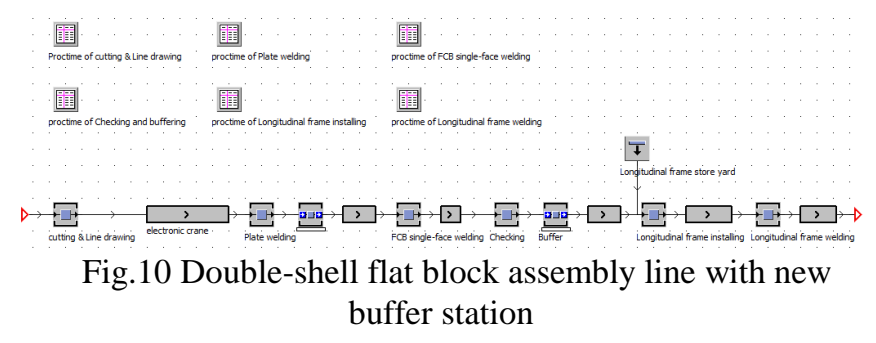

B. Analysis of experiments results

Table. 2 Test results of buffer experiment on assembly line A that based on floor pull-in method

\begin{tabular}{|c|c|c|c|}
\hline \multirow[t]{2}{*}{ Tests } & \multirow[t]{2}{*}{ Buffer size } & \multicolumn{2}{|c|}{ Blocking percentage $(\%)$} \\
\hline & & FCB welding & Checking \\
\hline 1 & 1 & 69.4 & 75.2 \\
\hline 2 & 2 & 65.5 & 73.5 \\
\hline 3 & 3 & 64.6 & 72.6 \\
\hline 4 & 4 & 60.9 & 68.7 \\
\hline 5 & 5 & 56.1 & 67.8 \\
\hline 6 & 6 & 54.2 & 64.0 \\
\hline 7 & 7 & 49.1 & 59.6 \\
\hline 8 & 8 & 47.1 & 57.2 \\
\hline 9 & 9 & 42.0 & 51.6 \\
\hline 10 & 10 & 40.5 & 47.8 \\
\hline 11 & 11 & 36.0 & 42.1 \\
\hline 12 & 12 & 31.5 & 39.9 \\
\hline 13 & 13 & 27.0 & 35.4 \\
\hline 14 & 14 & 23.5 & 30.6 \\
\hline 15 & 15 & 19.9 & 26.2 \\
\hline 16 & 16 & 16.2 & 22.5 \\
\hline 17 & 17 & 11.7 & 18.1 \\
\hline 18 & 18 & 7.9 & 14.4 \\
\hline 19 & 19 & 6.9 & 9.9 \\
\hline 20 & 20 & 5.7 & 6.1 \\
\hline 21 & 21 & 4.2 & 5.1 \\
\hline 22 & 22 & 2.6 & 3.9 \\
\hline 23 & 23 & 1.3 & 2.4 \\
\hline 24 & 24 & 1.3 & 0.8 \\
\hline 25 & 25 & 1.3 & 0 \\
\hline 26 & 26 & 1.3 & 0 \\
\hline 27 & 27 & 1.3 & 0 \\
\hline 28 & 28 & 1.3 & 0 \\
\hline 29 & 29 & 1.3 & 0 \\
\hline 30 & 30 & 1.3 & 0 \\
\hline
\end{tabular}

From Table. 2 above we can easily know that blocking percentage is decreasing as the volume of buffer station climbing. When volume larger than 25 blocking percentage of FCB welding station is in the lowest level which is nearly $1.3 \%$, meantime blocking of checking station can be completely diminished. Theoretically setting certain volume of buffer station after FCB welding station could also lower the blocking percentage of this working station. However, considering space of the flow shop, equipment, benefits and possibility this is not recommended. 


\section{CONCLUSION}

In this paper, we presented an idea that implement double-flat floor pull-in method in double-shell flat block assembly line and built a simulation model of double-shell flat floor assembly line by simulation software Plant Simulation and operated this model on the basis of double-flat floor pull-in method. By analysing the outcomes we found blocking situation on several stations. To avoid this problem we use DOE theory to conduct multiple experiments and find a way to change location of buffer stations which can solve this problem. By applying relocated buffer station the blocking situation is well solved.

\section{REFERENCES}

[1] Jaehun Park, Donha Lee, Joe Zhu. An integrated approach for ship block manufacturing process performance evaluation: Case from a Korean shipbuilding company. International Journal of Production Economics[J], 2014, 156:214-222

[2] Song Young-Joo, Lee Dong-Kun, Choe Sung-Won. A Simulation-Based Capacity Analysis of a Block-Assembly Process in ship Production Planning. Journal of the Society of Naval Architects of Korea [J], 2009, 46:01:1225-1143.

[3] Zhong Yu-guang, Xue Kai, Zhan Yong. Modeling and Analysis of panel hull block assembly system through timed colored Petri net. Marine Structures[J], 2011, 24:04:570-580.

[4] Yong-Kuk Jeong, SuHeon Ju, Huiqiang Shen. An analysis of shipyard spatial arrangement planning problems and a spatial arrangement algorithm considering free space and unplaced block. The International Journal of Advanced Manufacturing Technology[J],2018, 95:12, 4307-4325

[5] Seo Yoonho, Sheen Dongmok, Kim Taioun. Block assembly planning in shipbuilding using case-based reasoning. Expert Systems with Applications [J]. 2007 Vol.32 NO.1 P245-253 0957-4174.

[6] Kwangkook Lee, Jong Gye Shin \& Cheolho Ryu. Development of simulation-based production execution system in a shipyard: a case study for a panel block assembly shop, Production Planning \& Control: The Management of Operations[J], 2009, 20:8, 750-768

[7] N Fafandjel, RL Storch, D Kolich. Lean Methodology to Transform Shipbuilding Panel Assembly, Journal of Ship Production \& Design[J]. 2017.

Wang Yue, Professor, School of Naval Architecture \& Ocean Engineering, Jiangsu University of Science and Technology, Zhenjiang, Jiangsu, China.

HE Lei, Graduate student, School of Naval Architecture \& Ocean Engineering, Jiangsu University of Science and Technology, Zhenjiang, Jiangsu, China. 